\title{
SUSTENTABILIDADE AMBIENTAL E 0 REAPROVEITAMENTO DE GARRAFAS PETS NA PRODUÇÃO DE ENFEITES NATALINOS NA CIDADE DE PRATA-MG
}

\author{
Anaísa Filmiano Andrade Lopes ${ }^{1}$ \\ Diogo Sá da Silva Pompeu²
}

\begin{abstract}
RESUMO: O desenvolvimento industrial e o processo de urbanização fomentaram o uso de novas tecnologias e a produção em larga escala. A quantidade de recursos naturais explorados teve significativo aumento e como consequência, ampliou-se também a geração de resíduos sólidos urbanos, dentre eles, pode-se destacar o Pet - Politereftalato de Etileno. O reaproveitamento e a reciclagem de materiais dessa origem se caracterizam como medidas fomentadoras à sustentabilidade ambiental. A presente pesquisa tem como objetivo apresentar a estratégia utilizada na cidade de Prata - MG para o reaproveitamento de garrafas pets através da criação de enfeites natalinos, se caracterizando como estudo de caso. $O$ procedimento metodológico foi pautado na busca de informações e dados pertinentes em livros, artigos, dissertações, e em teses envolvendo a temática. Além do trabalho de campo na Prefeitura Municipal de Prata, especificamente na Secretaria de Meio Ambiente e no Centro de Múltiplo Uso do Prata - local responsável pela fabricação de enfeites natalinos e pelo armazenamento dos mesmos. Os resultados apontam que essa estratégia trouxe benefícios ambientais, dentre eles o aumento do tempo de vida útil do local de disposição final para onde as garrafas pets seriam direcionadas (aterro controlado), uma vez que a prefeitura da cidade deixou de encaminhar 54 toneladas de garrafas/ano. Conclui-se que a implantação do Centro de Múltiplo Uso, exerce a função que representa elementos caracterizados como potencialidades no cenário analisado, ou seja, suas ações são voltadas para a sustentabilidade ambiental, visto que o mesmo realiza o reaproveitamento e a reciclagem de materiais pets.
\end{abstract}

PALAVRAS-CHAVE: Sustentabilidade Ambiental. Reaproveitamento. PETS. Prata-MG.

\section{ENVIRONMENTAL SUSTAINABILITY AND THE REUSE OF PET BOTTLE'S PRODUCTION OF CHRISTMAS ORNAMENTS IN THE PRATA-MG CITY}

\author{
ABSTRACT: The industrial development and the urbanization process fostered the expansion \\ and use of new technologies and large-scale production. The amount of exploited natural

\footnotetext{
${ }^{1}$ Mestranda em Arquitetura e Urbanismo, Universidade Federal de Uberlândia - UFU. ana_isaandrade@hotmail.com

${ }^{2}$ Mestrando em Geografia, Universidade Federal de Uberlândia - UFU. diogo_geoufu@yahoo.com.br
} 
resources increased significantly and as a result also extended up the generation of municipal solid waste, among which we can highlight the Pet - polyethylene terephthalate. The reuse and recycling of materials that source are characterized as fueling measures environmental sustainability. This study aims to present the strategy used in the Prata City - MG for the reuse of plastic bottles by creating Christmas decorations, been characterized as a case study. The methodological procedure was guided in finding relevant information and data in books, technical manuals, articles, dissertations, and thesis involving the theme. In addition to the Prata in the City Hall fieldwork, specifically the Department of Environment and Multiple Use of Prata Centre - site responsible for making Christmas decorations and the storage thereof. The results show that this strategy has brought environmental benefits, including increased service life of the final disposal site where the plastic bottles would be directed (landfill), since the city government failed to send 54 tons of bottles / year. It is concluded that the implementation of the Multiple Use Center, plays a role representing elements characterized as potential in the analyzed scenario, so, their actions are geared towards environmental sustainability, since it performs the reuse and recycling of pets materials.

KEYWORDS: Environmental sustainability. Reuse. PETS. Prata-MG.

\title{
SOSTENIBILIDAD AMBIENTAL Y LA REUTILIZACIÓN DE BOTELLA PETS EN LAPRODUCIÓN DE ADORNOS DE NAVIDAD EM LA CIUDAD DE PRATA-MG
}

\begin{abstract}
RESUMEN: El desarrollo industrial y el proceso de urbanización fomentaron la expansión y uso de las nuevas tecnologías y la producción a gran escala. La cantidad de recursos naturales explotados aumentó significativamente y como resultado también se extendió la generación de residuos sólidos municipales, entre las que podemos destacar la botella Pet - tereftalato de polietileno. La reutilización y el reciclado destos materiales se caracterizan por ser fomentadora en las medidas de la sostenibilidad ambiental. Este estudio tiene como objetivo presentar la estrategia utilizada en la Ciudad de Prata - MG para la reutilización de botellas de plástico mediante la creación de decoraciones de Navidad, ha caracterizado como un estudio de caso. El procedimiento metodológico fue guiado en la búsqueda de información relevante y datos en libros, manuales técnicos, artículos, disertaciones y tesis que implica el tema. Además del trabajo de campo en el Ayuntamiento nel Departamento de Medio Ambiente y de Usos Múltiples del Centro de Prata - sitio responsable por hacer decoraciones de Navidad y el almacenamiento de los mismos. Los resultados muestran que esta estrategia ha generado beneficios ambientales, incluido el aumento de la vida útil del sitio de disposición final, donde se dirigirían las botellas de plástico (relleno sanitario), ya que el gobierno de la ciudad dejo de enviar 54 toneladas de botellas / año. Se concluye que la ejecución del Centro de Usos Múltiples, juega un papel que representa elementos caracterizados como potencial en el escenario analizado, es decir, sus acciones están orientadas a la sostenibilidad del medio ambiente, ya que se lleva a cabo la reutilización y el reciclaje de materiales pets .
\end{abstract}

PALABRAS-CLAVE: Sostenibilidad Ambiental. Reutilizar. PETS. Prata-MG.

\section{INTRODUÇÃO}

A industrialização promoveu a fabricação de bens não duráveis. As características e o volume dos resíduos gerados trazem consequências graves 


\section{ANAP $B_{\text {rasil }}$ \\ ISSN 1904-3240 \\ V. 8, n. 11}

para saúde pública e ambiental. Junto à industrialização, o processo de urbanização, aliado ao consumo crescente de produtos menos duráveis ou descartáveis, provocou um aumento do volume de resíduos gerados, bem como de sua concentração espacial (FINCO et. al., 2005).

A sustentabilidade ambiental surge como fundamento a um ambiente saudável para as gerações futuras, induzindo a pensar como os atores sociais poderiam retardar esse processo de descarte, dentre as medidas tem-se 0 reaproveitamento. Para Mano e Pacheco (2010), há muitas vantagens e benefícios para se reciclar ou reaproveitar um material, pois quando se reaproveita/recicla há menos poluição no ar, na água e no solo, há economia de energia elétrica e matéria-prima, além de melhorarias na limpeza da cidade.

Sem dúvida, os plásticos fazem parte da vida cotidiana do indivíduo; pode-se, nesse caso, citar o material PET (Politereftalato de Etileno). A origem da palavra plástico vem do grego plastokós, que significa: adequado à moldagem. Os plásticos são materiais formados pela união de grandes cadeias moleculares chamadas polímeros que, por sua vez, são formados por moléculas menores chamadas de monômeros. Os polímeros podem ser naturais, como: algodão, madeira, cabelos, látex, entre outros; e podem ser sintéticos, como o plástico, podendo ser obtidos através de reações químicas (RODRIGUES; FORMIGONI, 2009).

O material PET é, visivelmente, o mais encontrado nas garrafas de refrigerante. Esse plástico "alcançou espaço nos projetos ambientais devido ao seu material ser maleável, de fácil acesso, podendo ser trabalhado nas diversas classes sociais, e a facilidade de reutilização do material, podendo ser reutilizado desde o fundo da garrafa até a tampa" (SOUSA et. al., 2012), dessa forma, tudo pode ser aproveitado. Seguindo dados de Zaparolli (2007), cerca de $50 \%$ do PET brasileiro é reciclada. Leite (2003) cita que um dos problemas originados no descarte de materiais plásticos no Brasil é o espaço que ocupam nos aterros sanitários. Embora representem algo em torno de $10 \%$ do peso total do lixo, ocupam até $20 \%$ de seu volume, contribuindo para o aumento dos 


\section{ANAP $B_{\text {rasil }}$}

ISSN 1904-3240

V. 8, n. 11

custos de coleta, transporte e descarte final dos resíduos urbanos. Assim, algumas cidades buscam estratégias diferenciadas para o reaproveitamento dessas garrafas para minimizar os impactos que seriam causados no ambiente.

Diante desse contexto, a presente pesquisa objetiva apresentar a estratégia utilizada na cidade de Prata - MG para o reaproveitamento de garrafas PETS por meio da criação de enfeites natalinos.

A justificativa pela escolha da pesquisa deve-se à atualidade da temática, sendo que o acelerado crescimento populacional urbano e os novos padrões de vida essencialmente consumista imposto pela sociedade industrial, resultam no aumento da geração de resíduos sólidos. O manejo inadequado dos mesmos ocasiona problema ambiental e sanitário, pois provocam poluição do meio ambiente (poluição da atmosfera e, muitas vezes contaminação do solo e dos recursos hídricos) e, devido à proliferação de vetores propiciam o aparecimento de doenças, as quais devem ser enfrentadas como um sério problema de saúde pública (ALBERTIN, 2011).

Assim, analisar as estratégias que visam o reaproveitamento desses resíduos são medidas eficientes em prol da sustentabilidade ambiental, uma vez que visam a mínima degradação do meio ambiente.

\section{METODOLOGIA}

As técnicas de coleta de dados e informações, segundo Lakatos e Marconi (2003) e Salazar (2007), podem ser organizadas em dois grupos, sendo: o da documentação indireta e o da documentação direta. A documentação indireta envolve levantamentos de dados e informações por meio de pesquisa documental, bibliográfica e na internet. A documentação direta, por sua vez, "é a fase de levantamento de dados no próprio local onde os fenômenos ocorrem. Nessa etapa, os dados podem ser obtidos de duas maneiras: pesquisa de laboratório e pesquisa de campo" (SALAZAR, 2007, p. 


\section{ANAP

38). Desse modo, nesta pesquisa utilizaram-se procedimentos de documentação indireta e direta, conforme o organograma 01.

Organograma 01: Aspectos principais da Metodologia Empregada.

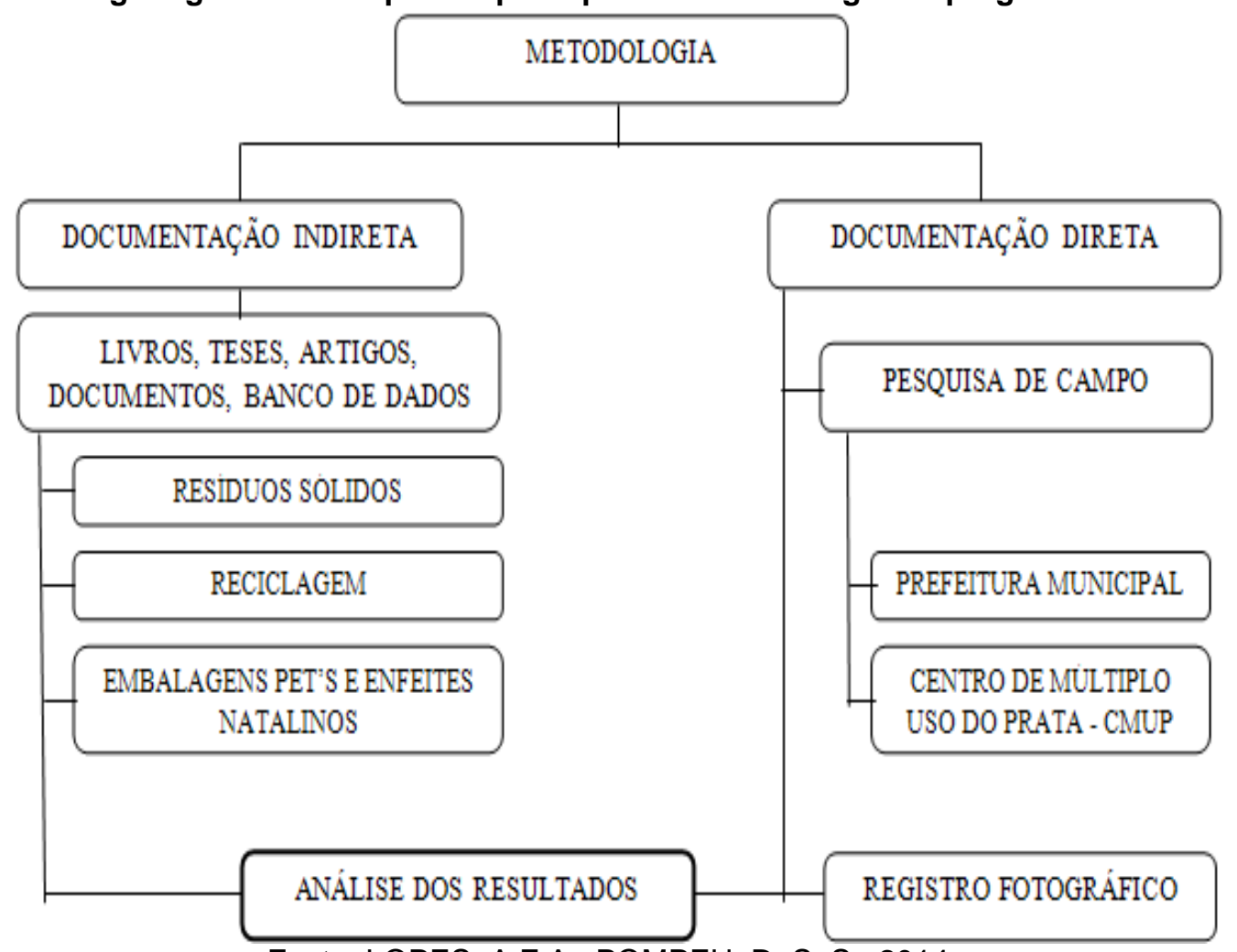

Fonte: LOPES, A.F.A.; POMPEU, D. S. S., 2014.

Especificamente, a documentação indireta foi realizada por meio de pesquisas em livros, manuais técnicos, artigos, dissertações, e em teses sobre as temáticas: resíduos sólidos, reciclagem, embalagens PETS e enfeites natalinos. Na documentação direta, foram realizados trabalhos de campo, por meio dos quais se efetuaram observações diretas, registros fotográficos e coleta de dados (obtidos através da gestão pública municipal, especificamente na Secretaria de Meio Ambiente e no Centro de Múltiplo Uso do Prata (CMUP), local responsável pela fabricação de enfeites de natal e pelo armazenamento dos mesmos. A visita de campo no CMUP da cidade foi fundamental para o levantamento de informações relevantes, sendo permitida por meio de uma 


\section{ANAP Brasil \\ ISSN 1904-3240 \\ V. 8, n. 11}

autorização concedida pelo secretário de meio ambiente da Prefeitura Municipal.

A pesquisa se caracteriza como estudo de caso, analisando a importância da criação de enfeites natalinos por meio do reaproveitamento de garrafas PETS na cidade de Prata - MG. Conforme Yin (2005) o estudo de caso representa a estratégia preferida quando o foco se encontra em fenômenos contemporâneos inseridos em algum contexto da vida real.

\section{RESULTADOS OBTIDOS}

O município de Prata é o maior em extensão territorial do Triângulo Mineiro/Alto Paranaíba, com $4.856 \mathrm{~km}^{2}$. Ele está situado no centro geográfico desta mesorregião geográfica (Figura 1).

Figura 1 - Localização do Município de Prata - MG na mesorregião geográfica Triângulo Mineiro/Alto Paranaíba, 2008.

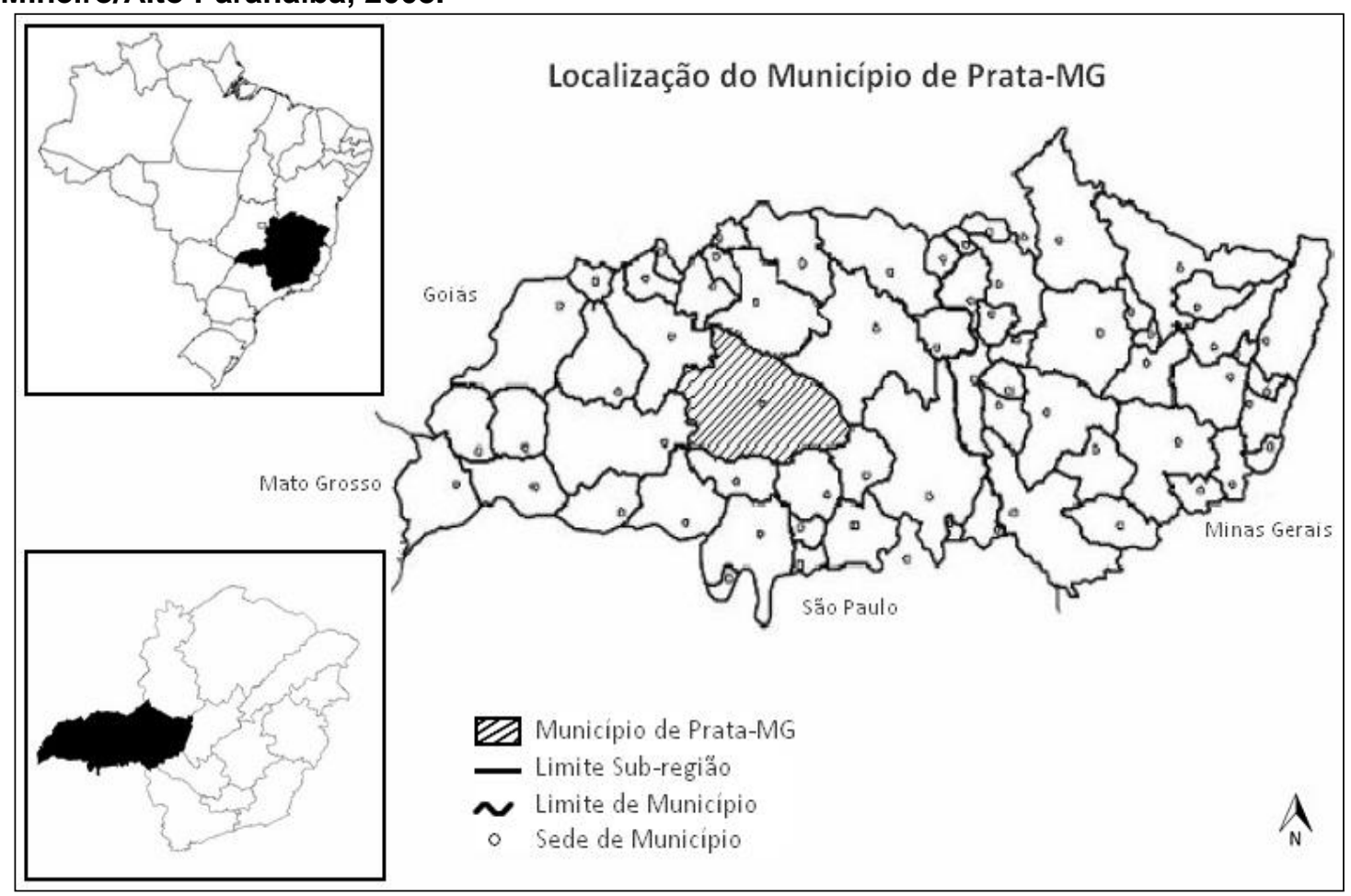

Fonte: FERREIRA, 2008. Adaptação: LOPES, A. F. A.; POMPEU, D. S. S., 2014. 


\section{ANAP Brasil $_{\text {ra }}$ \\ ISSN 1904-3240 \\ V. 8, n. 11}

\section{REVISTA}

De acordo com o último censo realizado pelo IBGE (2010), Prata possui uma população urbana de 19.381 habitantes e a população rural de 6.421 habitantes, totalizando 25.802 habitantes. A mesma não é diferente de outras cidades brasileiras, mesmo sendo de pequeno porte, apresentou uma forte expansão urbana desde as últimas décadas do século passado (NOVAES, 2013).

Segundo Ferreira (2008), nesta cidade, a indústria, o comércio e os serviços demonstram um típico quadro de economia que se assenta sobre uma base agrícola, na qual as atividades urbanas se desenvolvem principalmente como apoio às do setor primário.

O crescimento da população urbana e das atividades econômicas na cidade, bem como a mudança nos hábitos de consumo das pessoas acarreta 0 aumento na geração de resíduos sólidos. Consequentemente são maiores as demandas com relação aos serviços de gerenciamento dos mesmos.

O serviço público de coleta deste município, recolhe cerca de 12,1 toneladas de resíduos sólidos por dia, sendo, em média, 0,470 kg de resíduos por pessoa/dia (PMP, 2013), dentre eles, podemos destacar o material PET.

A gestão pública da cidade de Prata apresenta uma estratégia interessante quanto ao uso das garrafas PETS. No decorrer do ano as mesmas são coletadas por meio do programa de coleta seletiva nos bairros $\mathrm{e}$ destinadas a Usina de Reciclagem e Compostagem - URC. De acordo com Lopes e Melo (2014), o programa de coleta seletiva é composto pela coleta de porta em porta dos resíduos previamente separados pela população. O serviço de coleta dos materiais recicláveis é realizado em todos os bairros da cidade, sendo este trabalho executado por quinze cooperados da Cooperativa dos Agentes Ambientais de Prata (CAAP).

A CAAP realiza também a triagem dos resíduos coletados na cidade. Neste processo, os cooperados separam os resíduos orgânicos compostáveis, segregam e compactam os resíduos recicláveis conforme a natureza dos materiais e selecionam os rejeitos. Os resíduos recicláveis são vendidos para 


\section{ANAP $B_{\text {rasil }}$ \\ ISSN 1904-3240 \\ V. 8, n. 11}

REVISTA C I EN TÍFICA

2015

indústrias de reciclagem localizadas, principalmente, nos estados de São Paulo

e Goiás e o faturamento deste negócio é dividido entre os catadores (LOPES; MELO, 2014).

No final do ano há um incentivo ao reaproveitamento e a reciclagem por meio da produção de enfeites natalinos (figura 02). A cidade possui o Centro de Múltiplo Uso do Prata (CMUP), onde tem-se uma equipe de funcionários que produzem as decorações natalinas (figura 03). É neste local que os enfeites ficam condicionados antes de serem instalados na praça central - Praça XV de Novembro.

Figura 02: Decoração natalina (velas), praça Figura 03: Centro de Múltiplo Uso, Prata-MG. XV de novembro, Prata-MG.

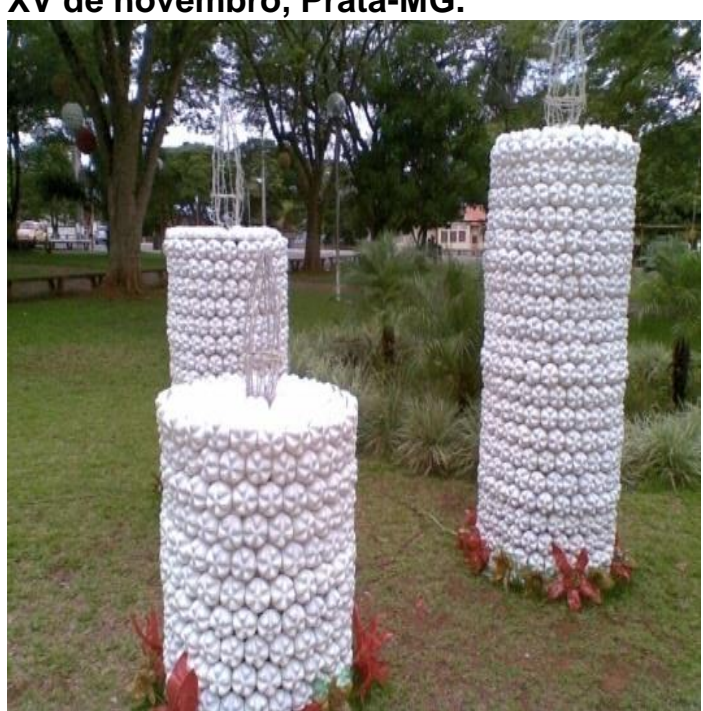

Fonte: LOPES, A. F. A, 2013.

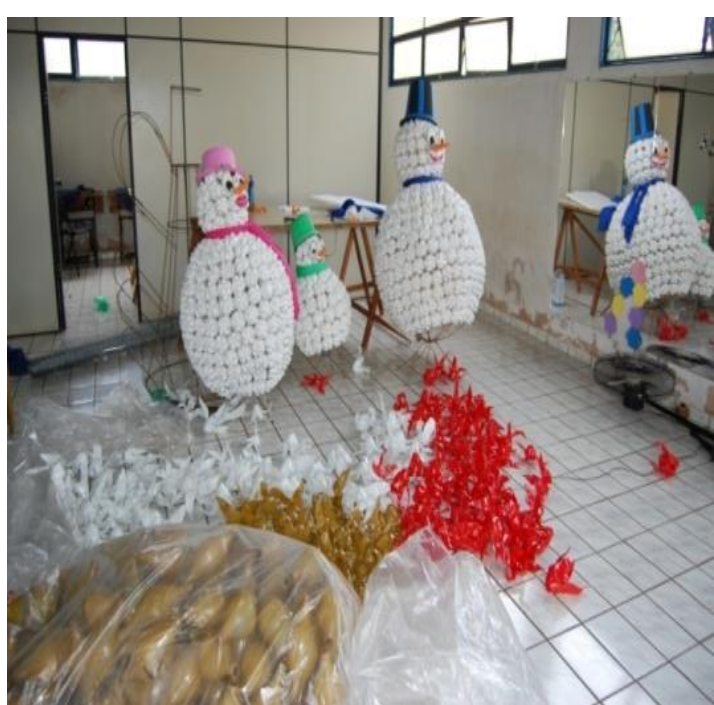

Fonte: LOPES, A. F. A, 2013.

$\mathrm{Na}$ cidade de Prata, o início da ideia e do projeto foi fomentada por um convidado (palestrante) que abordou a importância do reaproveitamento e da criação das decorações de natal, posteriormente os funcionários públicos seguiram com pesquisas na internet com intuito de elaborá-las.

Segundo o CMUP, como a ideia teve resultados favoráveis, os funcionários foram convidados a realizar palestras orientando a construção da decoração reciclável e reutilizável em outros locais. Por exemplo, em 2012 


\section{ANAP

foram convidados para realizar uma oficina de aprendizado em Campo FloridoMG.

De acordo com o CMUP, a árvore de natal da cidade, com quase 5 metros de altura, foi feita com mais de 7000 garrafas PETS picotadas, já nas asas de anjo foram utilizadas 600 garrafas para decoração (figura 04). As mesmas foram adquiridas por meio da própria sociedade, principalmente com o envolvimento dos alunos da rede municipal de ensino (CMUP, 2013).

Figura 04: Decoração natalina (anjo), praça XV de novembro, Prata-MG.

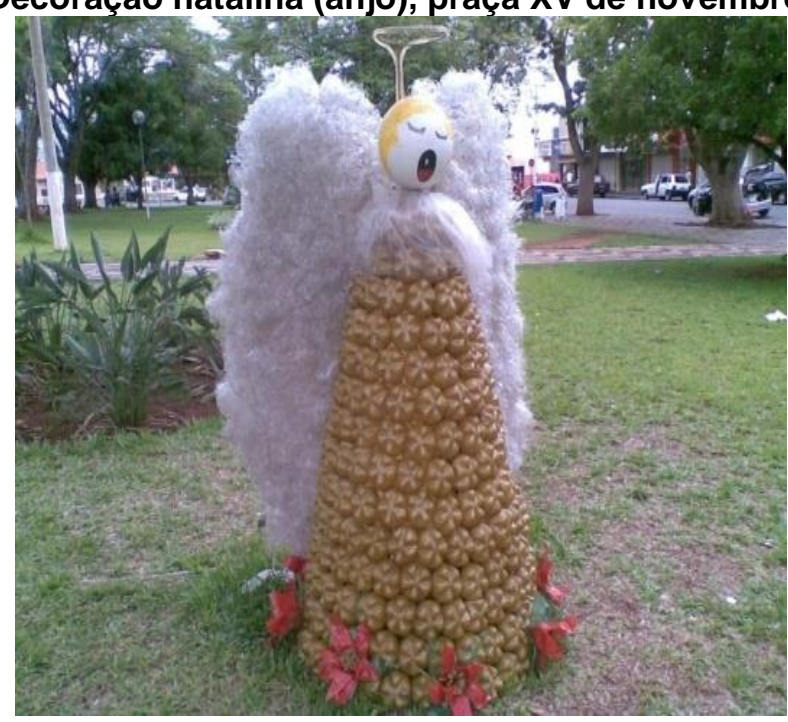

Fonte: LOPES, A. F. A, 2013.

Com essa ideia a prefeitura de Prata deixou de direcionar para o aterro cerca de 10 mil garrafas, equivalente a 54 toneladas (CMUP, 2013). Com essa atitude simples e de baixo custo, a prefeitura economizou recursos com a decoração natalina e com a coleta dos PETS nas ruas, uma vez que a população da cidade passou a direcionar as garrafas ao CMUP, favorecendo uma conscientização coletiva. As Figuras 05 e 06 mostram algumas das alternativas criadas, exemplo: casa do Papai Noel situada no centro da cidade (praça XV de Novembro) toda feita em garrafas. 


\section{ANAP $B_{\text {rasil }}$ \\ ISSN 1904-3240 \\ V. 8, n. 11}

\section{REVISTA C IENTIF I CA 2015}

Figura 05: Casa do Papai Noel, praça XV de Novembro, Prata-MG.

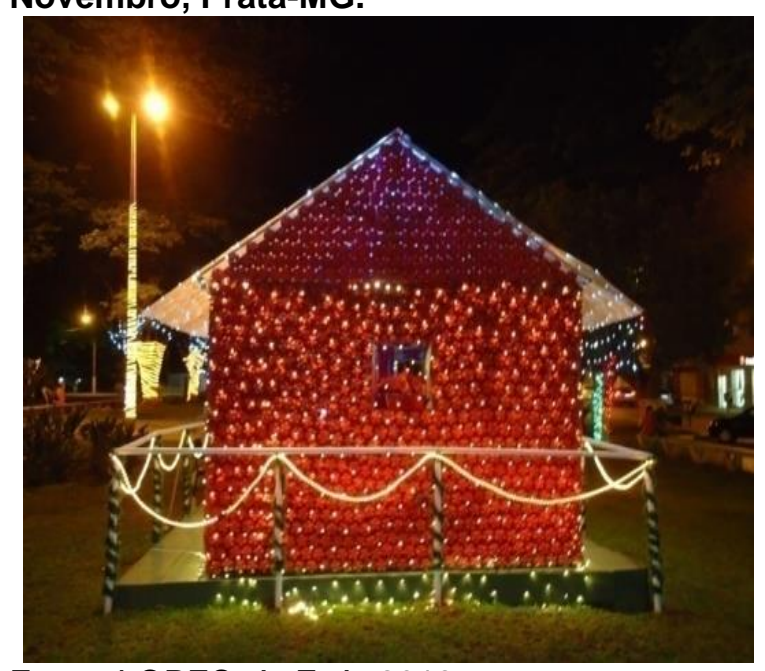

Fonte: LOPES, A. F. A, 2013.
Figura 06: Garrafas PET'S com piscas-piscas - casa do Papai Noel, Prata-MG.

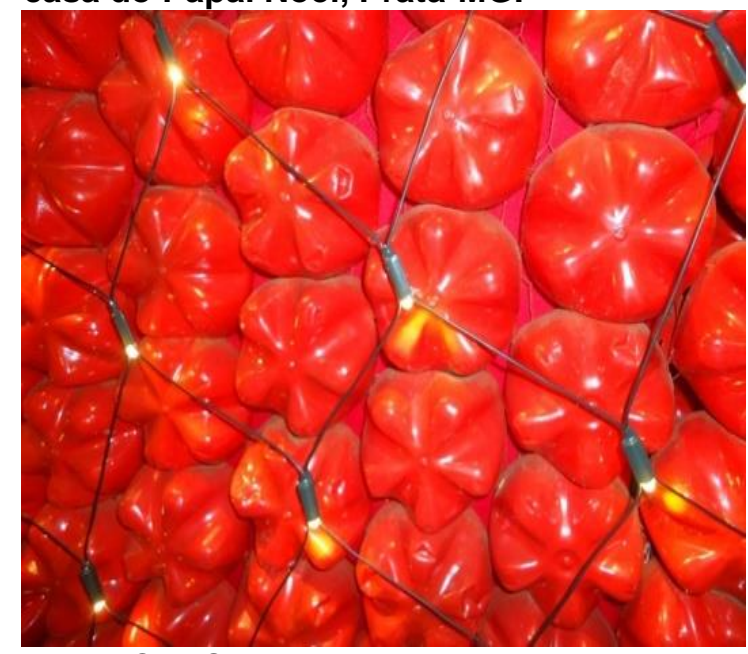

Fonte: LOPES, A. F. A, 2013.

A cidade utiliza há três anos os enfeites na praça XV de novembro, a ideia teve início em 2011 e prosseguiu nos anos 2012 e 2013, a cada ano os enfeites anteriores são reaproveitados, aperfeiçoados e novos são criados. Quando chega a noite aumenta o movimento de familiares no entorno da praça, é perceptível a admiração das pessoas e a beleza dos enfeites natalinos feitos com os materiais recicláveis. Essa atitude sustentável além de promover um convívio social harmônico, também aumentou o tempo de vida útil do local de disposição final para onde as garrafas PETS seriam direcionadas, sendo que a prefeitura da cidade deixou de encaminhar 54 toneladas de garrafas/ano ao aterro.

\section{CONCLUSÕES}

Um produto frequentemente utilizado pela população, independente de classe social, é a garrafa PET. Entretanto, é resistente à biodegradação, sendo resistente à radiação, calor, ar e água, mantendo as suas propriedades físicas e apresentando um tempo de decomposição longo, podendo causar sérios 


\section{ANAP

impactos ambientais. Assim, torna-se necessário o reaproveitamento, reciclagem e a destinação correta desses materiais.

Em Prata-MG, a implantação do Centro de Múltiplo Uso (CMUP), foi uma das ações importantes no que se refere à reciclagem na cidade em estudo. Esta instituição exerce função que representam elementos caracterizados como potencialidades no cenário analisado, ou seja, suas ações são voltadas para a sustentabilidade ambiental, visto que realiza 0 reaproveitamento e a reciclagem de materiais PETS.

A ideia de decoração natalina com materiais recicláveis está cada vez mais difundida. É uma alternativa que une criatividade, baixo custo e, principalmente, consciência ecológica. No Centro de Múltiplo Uso da cidade os funcionários são capacitados e os conhecimentos adquiridos, juntamente com a imaginação, possibilitam a transformação dos materiais em peças natalinas.

O CMUP contribui para a preservação dos recursos naturais e para a destinação ambientalmente correta de resíduos. A reciclagem vem também contribuindo para o aumento da vida útil da área de disposição final, pois com o reaproveitamento dessas garrafas, reduz-se 0 volume de resíduos encaminhados para o aterro municipal. Percebe-se que essa medida é fomentadora da sustentabilidade ambiental uma vez que o descarte indevido desse tipo de resíduo pode ocasionar escassez e esgotamento de recursos naturais, poluição do ar, da água, do solo, além de problemas de saúde pública devido à proliferação de vetores transmissíveis de doenças. Além dos benefícios ecológicos, a ideia também trouxe benefícios sociais, uma vez que a praça XV de Novembro passa a ser a noite um ponto de encontro, lazer e distração da população Pratense.

\section{REFERÊNCIAS}

ALBERTIN, Ricardo Massulo; Diagnóstico da Gestão dos Resíduos Sólidos Urbanos nos municípios da Bacia Hidrográfica do Rio do Índio - Estado do Paraná. Maringá, 2011. p. $50-51$. 


\section{ANAP

CMUP. Centro de Múltiplo uso do Prata. Dados obtidos através da visita realizada ao centro de múltiplo uso da cidade. Prata, 2013.

IBGE. Cidades,2010. Disponível em: <http://www.ibge.gov.br/cidadesat/topwindow.htm?1>. Acesso em: 28 de abril de 2014.

LAKATOS, E. M.; MARCONI, M. de A. Fundamentos de Metodologia Científica. 5. Ed. São Paulo: Atlas, 2003. p.234-251.

LEITE, P. R. Logística Reversa: meio ambiente e competitividade. São Paulo: Prentice Hall, 2003. $246 \mathrm{p}$.

LOPES, A. F. A. MELO, N.A. Gestão e Gerenciamento de Resíduos Sólidos Urbanos de Prata - MG: O Uso da Metodologia SWOT como Ferramenta de Análise. Uberlândia, 2014. No prelo.

FERREIRA, C. J. As Transformações Socioespaciais da Cidade e do Município de PrataMG. 70f. Monografia (Graduação em Geografia) Universidade Federal de Uberlândia, Uberlândia-MG, 2008.Disponível em:

http://www.geografiaememoria.ig.ufu.br/downloads/270_Jean_Carlos_Ferreira_2008pdf. Acesso em: 11 de agosto de 2015.

FINCO, Marcus Vinícius Alves; VALADARES, Marcelo de Brito;SILVA, Muriene Alves. Gestão de Resíduos Sólidos na Cidade de Palmas/TO: Contribuições ao mecanismo de desenvolvimento limpo (MDL). Universidade Federal do Tocantins, Palmas-TO, 2005, pp. 2.

MANO, Eloisa Biasotto; PACHECO, Élen B. A. V.; BONELLI, Cláudia M. C. Meio Ambiente Poluição e Reciclagem. $2^{\circ}$ Ed. 2010, Edgard Blücher Ltda., pag. 114.

Novaes, T. G. Guia do município de Prata-MG. Prata-MG, 2013.

PMP, Prefeitura Municipal de Prata. Dados obtidos através da visita realizada na prefeitura.

Prata, 2013.

PMP, Prefeitura Municipal de Prata. Dados obtidos através da visita realizada ao secretário do Meio Ambiente. Prata, 2013.

RODRIGUES,Ê.F. FORMIGONI, A. A Busca pela Sustentabilidade do Pet, através da sustentabilidade da Cadeia de Suprimentos. UNESP - Faculdade de Engenharia de Guaratinguetá, São Paulo, 2009.

SALAZAR, L. V. Fundamentos de Metodologia Científica para Elaboração de Trabalhos Acadêmicos: material para fins didáticos. Uberlândia, 2007.

SOUSA, T. K. A. de; MOURA, J. M. de; FERNANDES, A. T.s.III Congresso Brasileiro de Gestão Ambiental, 2012. Reutilização de Pet como Prática de Educação Ambiental na creche Municipal Wilmon Ferreira de Souza - Bairro Três Barras, Cuiabá - MT. Disponível em: http://www.ibeas.org.br/congresso/Trabalhos2012/VII-003.pdf. Acesso em: 10 de agosto de 2015.

YIN, R. K. Estudo de caso: planejamento e métodos. 3ed. Porto Alegre: Bookman, 2005.

ZAPAROLLI, D.PET Reciclado em Embalagens para Alimentos Gera Novas Perspectivas. RJ,2007. 